Case Report

\title{
LAPAROSCOPIC ADRENALECTOMY IN PEDIATRIC PATIENTS - A REPORT OF 2 CASES.
}

\author{
Sandeep B. Rai ${ }^{1}$, Rajesh Ballal', Raghu Shankar ${ }^{3}$ \\ ${ }^{1}$ Professor and Head, ${ }^{3}$ Associate Professor, Pediatric Surgery, ${ }^{3}$ Professor and head, Department of Surgery, \\ Justice K.S. Hedge Medical College, NITTE University, M angalore - 575018. \\ Correspondence : \\ Raghu Shankar \\ Associate Professor, Pediatric Surgery, Justice K.S. Hegde charitable super speciality hospital, \\ Deralakatte, Mangalore - 575 018, Karnataka. \\ Phone : +918242204471 E-mail : drraghubhat@yahoo.com
}

\section{Abstract:}

Reporting on the laparoscopic technique for adrenal disease in children and adolescents has been limited. Laparoscopic adrenalectomy are been performed in selected patients in centers with advanced laparoscopic expertise. Here we report two cases of laparoscopic adrenalectomy done for functional adrenal tumors in pediatric patients.

Keywords: Laparoscopic adrenalectomy, feminizing adrenal tumor, Cushing's adenoma.

\section{Introduction:}

The adrenal gland is considered suitable for laparoscopic resection because of its relatively small size and retroperitoneal location. Laparoscopic adrenalectomy (LA) has become the standard procedure for benign adrenal masses in adults. Despite multiple studies in the adults,

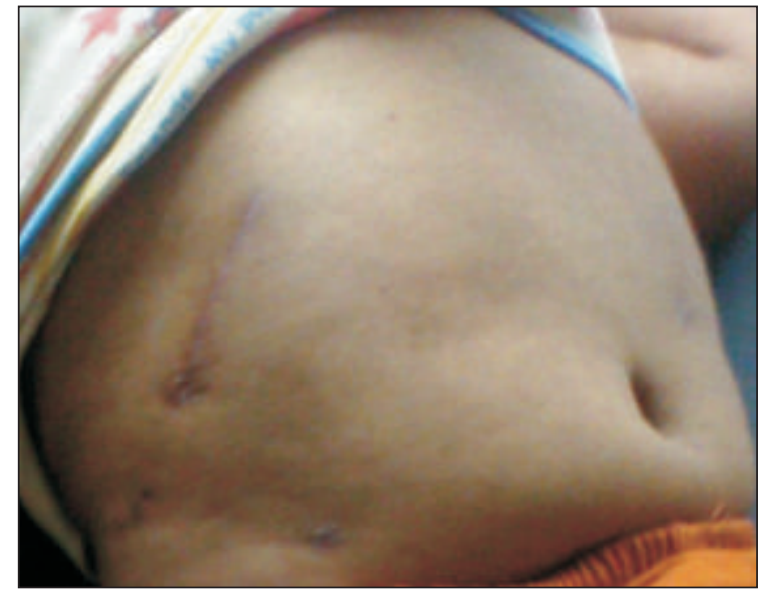

Fig 1. Port sites and the incision

\begin{tabular}{|c|}
\hline Access this article online \\
\hline Quick Response Code \\
\hline
\end{tabular}

only a few reports describe the outcome of this technique in pediatric patients. Infrequent indications for adrenalectomy in children and small body habitus have contributed to the delay in adopting $L A$ in

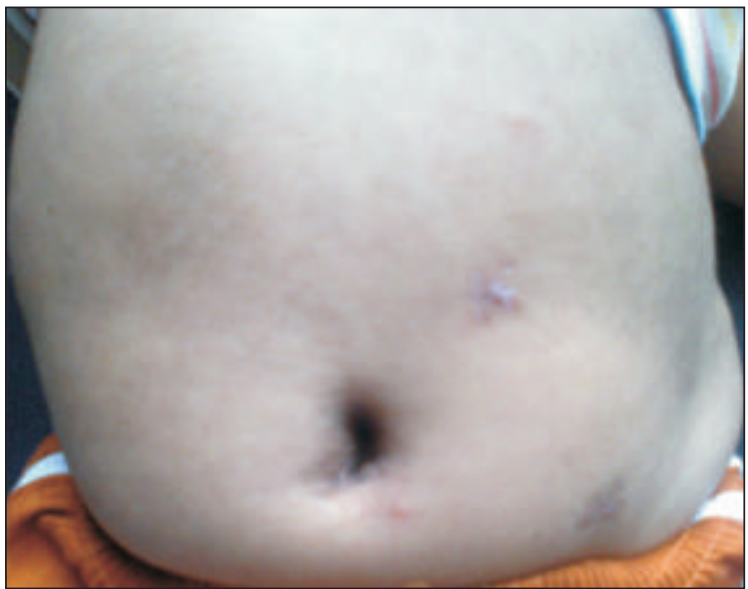

Fig 2. Port sites

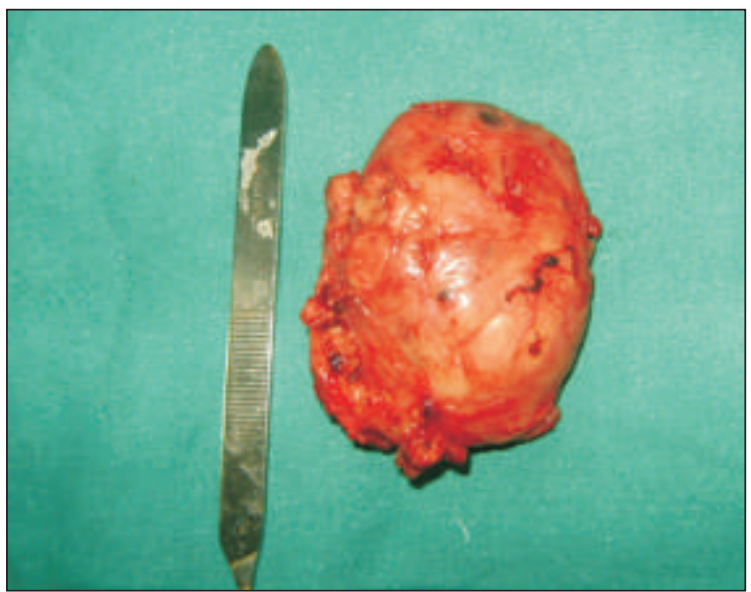

Fig.3 Cushing's adenoma

pediatric patients. Also, neuroblastoma is the most common adrenal lesion in this age group and this lesion is 
infiltrative and invasive in nature. As laparoscopic skills have developed in the pediatric surgical community, laparoscopic adrenalectomies are being performed for selected pediatric patients. However, this procedure is been performed in centers with advanced laparoscopic expertise. We report two cases of adrenal tumors in pediatric patients who were managed with laparoscopic adrenalectomy in our institution.

\section{Case report :}

Case 1 : A six year old male child was referred with gynecomastia of six months duration. Child had been evaluated elsewhere and was receiving systemic steroids for the past three weeks. Examination revealed cushiongoid facies with bilateral gynecomastia. Other systemic examination findings were within normal limits. The size of the penis was normal for the age and bilateral testes were normal. Serum estradiol was found elevated three times the normal value, and serum cortisol level was normal. Abdominal sonology showed a $3.5 \mathrm{~cm} \times 2.6 \mathrm{~cm}$ mass lesion in the right adrenal gland with specks of calcification. CT abdomen showed a well encapsulated right adrenal tumor. Transperitoneal laparoscopic adrenalectomy was done.. Post -op recovery was uneventful. As the histopathology revealed malignant adrenocortical feminizing tumor. Child received chemotherapy post operatively.

Case 2: An eleven month old male child presented with excessive weight gain and excessive body hair. On examination, child had cushingoid features, hirsutism and weighed $11 \mathrm{kgs}$. However, there were no features of precocious puberty. Child had borderline hypertension. Systemic examination including abdominal, respiratory, cardiovascular and central nervous system did not reveal any abnormality. Serum cortisol levels were found to be grossly elevated with the value of 36.14 microgram/dl (5.716.6). Random sugar was $97 \mathrm{mg} / \mathrm{dl}$; creatinine, sodium and potassium levels of serum were normal. Contrast enhanced CT of the abdomen showed a well-defined heterogeneously enhancing, $4.3 \mathrm{~cm} \times 3.7 \mathrm{~cm}$ soft tissue density lesion arising from the right adrenal. There was no evidence of local invasion. A diagnosis of Right side functional adrenal tumor was made. Child was taken up for laparoscopic adrenalectomy. The mass was noted to be of about $6 \times 5 \mathrm{~cm}$, well encapsulated and compressing the IVC.
However, the superior margin could not be defined well. Hence, keeping the possibility of malignancy, a biopsy was taken. Histopathology showed a benign adrenal tumor, Cushing's adenoma. And so, in the next sitting a week later, transperitoneal laparoscopic adrenalectomy was carried out. Recovery was uneventful. Child was started on oral liquids the next day and progressed to full diet on the subsequent day. The final histopathology revealed an intermediate grade adrenocortical tumor.

\section{Technique:}

For $L A$, the patient is placed in the $90^{\circ}$ lateral decubitus position with the operative side up.

A bolster is placed underneath the lumbar region and the table flexed maximally to create the largest possible distance between the costal margin and the iliac crest. Ports were introduced at the sites shown in the figure $1 \& 2$. Adrenal vein was seen only after initial dissection of the mass from lateral, inferior and superior borders using the harmonic dissector. Adrenal vein was coagulated with the harmonic dissector blades. The remaining gland was freed by circumferential dissection. In the first case at the completion of laparoscopic dissection, the hemostasis achieved was in doubt. Hence, to ensure absolute hemostasis, a small subcostal incision was put which also served to retrieve the specimen. Similarly, in the second case the small subcostal incision was used to deliver the specimen (fig 3 ).

\section{Discussion:}

The laparoscopic approach for excision of adrenal gland was first described by Gagner et al in 1992. ${ }^{1}$ Laparoscopic adrenalectomy has become the gold standard for removing adrenal lesions of almost any pathology in adults. ${ }^{2,3}$ Despite infrequent cases in the pediatric population, the wide applicability of laparoscopy to multiple pediatric surgeries has enabled pediatric surgeons with necessary skill and equipment to perform laparoscopic adrenalectomies. Potential benefits of laparoscopic adrenalectomy are similar to those of other minimally invasive procedures, including decreased postoperative pain, shorter hospitalization, and faster return to normal activity.

The principal approaches described for $\mathrm{L} A$ are the transperitoneal and the retroperitoneal approach. The 
lateral transperitoneal approach offers a more efficient working space and excellent exposure. ${ }^{4}$ It has been advocated by many to be the preferred approach. ${ }^{5}$

The indications for adrenal surgery differ in adults and children. Literature reveals neuroblastic tumours to be the most common adrenal lesions, requiring excision in the pediatric age group. ${ }^{6}$ The safety and feasibility of LA for all stages of neuroblastomas in pediatric patients (including infants) has been documented in earlier studies., ${ }^{5,6}$ Although no absolute contraindications to LA has been clearly identified in adults, open approach has been preferred in malignancies with metastatic nodes in periaortic area and close to the bladder. However, in children, LA has even been suggested as an option for residual tumours that have a favourable cytoreductive response to chemotherapy?. Rather than stating an absolute contraindicated mass size for pediatric patients, the size of the tumor may limit the laparoscopic approach when evaluated individually relative to the size of the

\section{Reference:}

1. Gagner M , LacorixA, Bolte E. Laparoscopic adrenalectomy in Cushing's syndrome and pheochromocytoma. N Engl J M ed 1992;327:1033.

2. Vargas HI, Kavoussi LR, Bartlett DL, Wagner JR, Venzon DJ, Fraker DL, et al.. Laparoscopic adrenalectomy: A new standard of care. Urology 1997;49:673-8.

3. Gil-Cardenas A, Cordon C, Gamino R, Rull JA, Gomez-Perez F, Pantoja JP, et al.. Laparoscopic adrenalectomy: Lessons learned from an initial series of 100 patients. Surg Endosc 2008;22: 991-4.

4. Suzuki K, Kageyama S, Hirano $Y$, et al: Comparison of 3 surgical approaches to laparoscopic adrenalectomy: A nonrandomized, background matched analysis. J Urol 166:437-443, 2001

5. Miller K.A, Albanese.C, Harrison.M, Farmer.D, Ostlie D.J, Gittes G, Holcomb G.W III. Experience with laparoscopic adrenalectomy in pediatric patients. J Pediatr Surg 2002 37:979-982.

6. Skarsgard ED, Albanese CT. The safety and efficacy of laparoscopic adrenalectomy in children. Arch Surg 2005;140:905-8

7. Kadamba P, Habib Z, Rossi L. Experience with laparoscopic adrenalectomy in children.J Pediatr Surg 2004;39:764-7

8. Gagner M, Pomp A, Heniford BT, et al: Laparoscopic adrenalectomy:Lessons learned from 100 consecutive procedures. Ann Surg 226:238-247, 1997 patient ${ }^{5}$. The surgeon's experience also should be considered in these cases. Relative contraindications include uncorrectable coagulopathy and previous trauma in the region of spleen and kidney ${ }^{8}$.

\section{Conclusion:}

Laparoscopic adrenalectomy can be performed safely and effectively with a short hospital stay and minimal blood loss in pediatric patients. Patient selection for laparoscopy is crucial and is dependent on the child's body habitus, as well as the experience of the surgical and anaesthetic teams. A low threshold for open conversion in the early phase of the learning curve is recommended.

\section{Acknowledgment:}

Our sincere thanks to department of pediatrics and department of anaesthesia, without whom the clinical course would not have been a smooth one. Also, thanking the nursing staff of the pediatric ward. 